\title{
Effect of Aspirin on C- Reactive Protein as a Marker of Risk in Unstable Angina
}

\author{
Lincy Joseph $^{1}$, Mathew George ${ }^{2}$, Bhagya Krishnan ${ }^{2 *}$ \\ ${ }^{1}$ Department of Pharmaceutical Chemistry, Pushpagiri College of Pharmacy, Thiruvalla-689107, Kerala, \\ India \\ ${ }^{2}$ Department of Pharmacology, Pushpagiri College of Pharmacy Thiruvalla-689107, Kerala, India
}

\begin{abstract}
Angina, is the term used for chest pain caused by reduced blood flow to the heart muscle. Angina is typically described as squeezing, pressure, heaviness, tightness or pain in chest. Aspirin, also known as acetyl salicylic acid (ASA) is a medication used to treat pain, fever and inflammation. Aspirin is also used long term to help prevent heart attacks, strokes and blood clots in people at high risk. Elevated levels of $C$ - reactive protein, a marker for systemic inflammation, have been found among individuals with stable and unstable angina. Elevated circulating levels of $C$-reactive protein, an inflammatory marker, increase the risk of thrombotic cardiovascular diseases such as myocardial infarction.
\end{abstract}

Keywords: Unstable angina, Aspirin, C-reactive protein, Cardiovascular diseases.

\section{Introduction}

Angina, is the term used for chest pain caused by reduced blood flow to the heart muscle. Angina is typically described as squeezing, pressure, heaviness, tightness or pain in chest. Angina can be classified in to two types, stable angina and unstable angina. Unstable angina or sometimes referred to as acute coronary syndrome causes unexpected chest pain, and usually occurs while resting. ${ }^{[1]}$ The most common cause is reduced blood flow to the heart muscle because the coronary arteries are narrowed by atherosclerosis which can rupture causing injury to the coronary blood vessels resulting in blood clotting which blocks the flow of blood to the heart muscle. It may get worse overtime and can lead to a heart attack. ${ }^{[2]}$

Aspirin is also used long term to help prevent heart attacks, strokes and blood clots in people at high risk. Aspirin is widely used either alone or combination with other anti platelet agents to prevent blood clots from forming in arteries. Aspirin is prescribed in moderate doses (160-325 mg/day) to patients who are having unstable angina to prevent heart attacks and improve survival. The principal goal of therapy for unstable angina is to restore normal blood supply to the heart. Aspirin reduce the formation of blood clots in the coronary arteries and restore normal blood flow. ${ }^{[3]}$

Elevated levels of c- reactive protein, a marker for systemic inflammation, have been found among individuals with stable and unstable angina who are at risk for future myocardial infarction or sudden death. Elevated circulating levels of C- reactive protein, an inflammatory marker, increase the risk of thrombotic cardiovascular diseases such as myocardial infarction.

Sunitha V Ittaman ${ }^{[4]}$ et.al.(2013) conducted a study on "The role of aspirin in the prevention of cardiovascular disease." In this review they described the seminal trials of aspirin use in the context of current guidelines, discuss factors that may influence the effectiveness of aspirin therapy for cardiovascular disease prevention, and briefly examine patterns of use. The body of evidence supports a role for aspirin in both secondary and primary prevention of cardiovascular events in selected population groups, but practice patterns may be suboptimal. As a simple and inexpensive prophylactic measure for cardiovascular disease, aspirin use should be carefully considered in all at- risk adult patients, and further measures, including patient education, are necessary to ensure its proper use.

Michelle A Albert ${ }^{[5]}$ et.al (2001) conducted a study on "effect of statin therapy on C- reactive protein levels." To test the hypothesis that pravastatin as anti-inflammatory effects as evidenced by $\mathrm{C}$ - reactive protein reduction. This is a community based, prospective, randomised, double blind trial including 1702 men and women with no prior history of cardiovascular disease (primary prevention cohort) and open label study including 1182 patients with no cardiovascular disease (secondary prevention cohort) who provided at least baseline and 12-week blood samples. Main outcome measure was change in C- reactive protein levels from baseline to 24 weeks. This prospective trial, pravastatin reduced C- reactive protein level at both 12 and 24 weeks in a largely LDL-C- independent manner. These data provide evidence that statins may have antiinflammatory effects in addition to lipid lowering effects.

Mark Feldman ${ }^{[6]}$ et.al. (2001) conducted a study of" effects of low dose aspirin on serum C- reactive protein and thromboxane B2 concentrations: a placebo controlled study using a highly sensitive C- reactive protein 
assay." Elevated circulating levels of C- reactive protein, an inflammatory marker, increase the risk of thrombotic cardiovascular diseases such as myocardial infarction. Moreover, low dose aspirin therapy has been reported to be more effective in preventing MI in men with higher C- reactive protein levels than it is in those with lower levels, raising the possibility that aspirin prevent thrombosis by reducing vascular inflammation. Effects of aspirin (81 mg every day or 325,81 or $40 \mathrm{mg}$ every- third-day given for 31 days) on serum Creactive protein, using a highly sensitive assay, and on serum platelet-cyclo-oxygenase (COX)-1- derived thromboxane (TX) B2 concentrations were studied simultaneously in 57 healthy volunteers. Low dose of aspirin that markedly inhibit platelet COX-1 activity, as manifested by a profound decline in platelet- derived serum TX $\mathrm{B} 2$ concentrations, have no detectable effect on serum $\mathrm{C}$ - reactive protein levels in healthy men and women.

Ross R et.al. ${ }^{[7]}(\mathbf{1 9 9 9})$ conducted a study on "Atherosclerosis is an inflammatory disease." Because high plasma concentrations of cholesterol, in particular those of low-density lipoprotein (LDL) cholesterol, are one of the principal risk factors for atherosclerosis, ${ }^{1}$ the process of atherogenesis has been considered by many to consist largely of the accumulation of lipids within the artery wall; however, it is much more than that. Despite changes in lifestyle and the use of new pharmacologic approaches to lower plasma cholesterol concentrations, cardiovascular disease continues to be the principal cause of death.

Mary Cushman ${ }^{[8]}$ et.al. (1997) conducted a study on "Plasma concentration of C- reactive protein and risk of developing peripheral vascular disease." Among apparently healthy men, elevated levels of C-reactive protein (CRP), a marker for systemic inflammation, predict risk of myocardial infarction and thromboembolic stroke. Whether increased levels of $\mathrm{C}$ - reactive protein are also associated with the development of symptomatic peripheral arterial disease is unknown. Using a prospective, nested, case- control design, they measured baseline levels of C- reactive protrein in 144 apparently healthy men participating in the physician's health study who subsequently developed symptomatic PAD and in an equal number of control subjects matched on the basis of age and smoking habit who remained free of vascular disease during a follow- up period of 60 months. These prospective data indicate that among apparently healthy men, baseline levels of CRP predict future risk of developing symptomatic PAD and thus provide further support for the hypothesis that chronic inflammation is important in the pathogenesis of atherothrombosis.

Paul M Ridker ${ }^{[9]}$ et.al (1997)conducted a study on "Inflammation, aspirin, and the risk of cardiovascular disease in apparently healthy men."Inflammation important in the pathogenesis of atherothrombosis. Inflammation increases the risk of a first thrombotic event and treatment with aspirin reduces the risk. They measured 543 healthy men. Subjects were randomly assigned to receive aspirin or placebo at the beginning of the trial. The baseline plasma concentration of $\mathrm{C}$ - reactive protein predicts the risk of future myocardial infarction and stroke.

\section{Conclusion}

The current review shows the study to be conducted in order to study the effect of aspirin on Creactive protein as a marker on risk in unstable angina. Administration of aspirin reduces the level of C- reactive protein. Elevated circulating levels of $\mathrm{C}$ - reactive protein, an inflammatory marker, increase the risk of thrombotic cardiovascular diseases such as myocardial infarction.

\section{References}

[1]. Liuzzo G, Biasucci L.M, Gallimore J. R, The prognostic value of c-reactive protein and serum amyloid A protein in severe unstable angina, N Engl. J med 1994: 417-424.

[2]. Rebuzzi A.G, Quaranta G, Liuzzzo G, instrumental prognosis of serum levels of troponin T and crp on admission in patients with unstable angina AM J cardiol 82 1998:715-719.

[3]. Morrow D. A, Rifai N, Antman E: crp is aa potent predictor of mortality independently of and in combination with troponin T in acute coronary syndromes, L TIMI 11 A sub study J. AM Coll cardiol 31 1998:14601465.

[4]. Sunitha V Ittaman, MD, Jeffrey J Vanvomer, PhD, Sherief H Rezkalla, MD, "The role of aspirin in the prevention of cardiovascular disease." Clin Med Res 2014 Dec 12(3-4):147-154.

[5]. Michelle A Albert, MD, Ellie Danielson, MIA, Nader Rifai, PhD, Paul M Ridker,MD, "Effect of statin therapy on C- reactive protein levels.” JAMA, July 2001-vol 286, no.1

[6]. Mark Feldman, IshwarlalJialal, SrideviDevaraj, Byron Cryer. "Effects of low dose aspirin on serum C- reactive protein and thromboxane B2 concentrations: a placebo controlled study using a highly sensitive C- reactive protein assay." J Am Cardiol. 2001:37(8):2036-2041.

[7]. Ross R. Atherosclerosis an inflammatory disease N Engl J med 1999:340:115-126.

[8]. 8.Mark Feldman, IshwarlalJialal, SrideviDevaraj, Byron Cryer. "Effects of low dose aspirin on serum C- reactive protein and thromboxane B2 concentrations: a placebo controlled study using a highly sensitive C- reactive protein assay." J Am Cardiol. 2001:37(8):2036-2041.

[9]. Paul M Ridker, Mary Cushman , Meir J Stampfer, Russell P Trasy, Charles H Hennekens “"'Inflammation, aspirin, and the risk of cardiovascular disease in apparently healthy men.” The New England Journal of Medicine Vol 336, April 31997 No 14. 